\title{
Enteroliths Masquerading as Urinary Bladider Stone
}

\section{Tanweer Karim, Subhajeet Dey}

Department of Surgery, ESIPGIMSR, Basaidarapur, New Delhi- 110015 , India.

\section{Abstract:}

Enterolith or concretion in the bowel is rare entity in humans. It is found to be associated with various diseases of intestine but its presentation mimicking urinary bladder calculi on radiograph makes it interesting. A 38 year old patient presented with lower abdominal pain and dysuria. Plain radiograph of abdomen was showing radiopaque densities in pelvis falling in close proximity of urinary bladder. However further investigation revealed them to be in the small intestine proximal to a narrow segment, possibly a stricture. Patient underwent laparotomy with resection of affected bowel segment and end to end anastomosis. Histopathology report showed it to be a nonspecific inflammatory stricture of small bowel with enteroliths.

Key words: Abdominal Pain, Constriction, Dysuria, Intestinal Obstruction, Urinary Bladder Calculi.

\section{Introduction}

An enterolith is a mineral concretion or stone formed around a nidus in the gastrointestinal system. Stasis is thought to be an important factor in both the conditions. It is usually composed of calcium phosphate and calcium carbonate. Presence of choleic Acid, deoxycholic acid and cholic acid has been found in some cases. Enteroliths have been reported in bowel affected by tuberculosis, strictures [1] and diverticula of small bowel including Meckel's diverticulum [2], ulcerative colitis, Crohn's disease, carcinoid tumor, post-traumatic or post-surgical stricture. Enteroliths are mostly result of sub-acute or chronic intestinal obstruction rather than cause of obstruction itself. Calculus disease of gastrointestinal tracts may remain asymptomatic till a long period of time. However, any complications that do occur are severe and serious in nature and more often than not require a surgical intervention. Most human enteroliths are radiolucent on plain $X$-ray. Ultrasound and with or without Computerised Tomography helps in clinching the diagnosis.

\section{Case Report}

A 38 year old male patient was being investigated for complaints of intermittent spasmodic pain of lower abdomen with dysuria since last one and half years. There was no history of fever or hematuria. He was treated for abdominal tuberculosis around 12 years back. His hematological, biochemical parameters and urine analysis were all within normal limits. X-ray of KUB revealed multiple radio-opaque shadows in the pelvic region [Fig.1]. The radio-opaque shadows were not seen on

\section{Corresponding Author: Dr. Tanweer Karim}

Email: karimtanweer@yahoo.co.in

Received: July 22, 2015 | Accepted: October 8, 2015 | Published Online: October 30, 2015

This is an Open Access article distributed under the terms of the Creative Commons Attribution License (creativecommons.org/licenses/by/3.0)

Conflict of interest: None declared | Source of funding: Nil | DOl: http://dx.doi.org/10.17659/01.2015.0120 
subsequent ultrasonography of abdomen but gave additional findings of mesenteric lymphadenopathy with mild ascites. This is the time we started thinking of enteroliths and a contrast CT scan of the abdomen was sought, which revealed ileal stricture with proximal dilatation and no mention of the radio-opaque shadows. There was no evidence of lymphadenopathy or ascites on CT scan of abdomen. However, a non-contrast screening CT of the abdomen revealed large enteroliths in the distal ileum [Fig.2] with possibility of stricture in ileum.

The patient underwent laparotomy via lower midline incision under general anesthesia. On exploration the patient was found to have multiple strictures of the proximal ileum with palpable enteroliths within. He underwent resection and anastomosis of the affected ileal loop along with the enteroliths [Fig.3]. There were no ascites, lymphadenopathy, diverticula, tubercles or adhesions or bands and gall bladder was normal. Post-operative period was uneventful and patient was discharged on $5^{\text {th }}$ post-operative day.

Histopathology of the resected ileum revealed three strictures along with two ulcers of size $3 \times 2 \mathrm{~cm}$ and $2.5 \times 1 \mathrm{~cm}$ at 7 and $9 \mathrm{~cm}$ from resected ends respectively. There was no evidence of granulomatous disease or malignancy. Patient is asymptomatic at one month follow up.

\section{Discussion}

An enterolith, first described in 1915 [3], is mixed concretion usually formed in gastrointestinal tract of equines, rarely in humans. In humans usually three types of enteroliths are found containing bile acids, calcium oxalate, or phosphate [4]. Proximal small bowel enteroliths are usually composed of bile acids whereas distal small bowel (ileal) enteroliths contain calcium. It is postulated that diverticula provide the more acidic environment necessary for choleic acid precipitation and stone formation

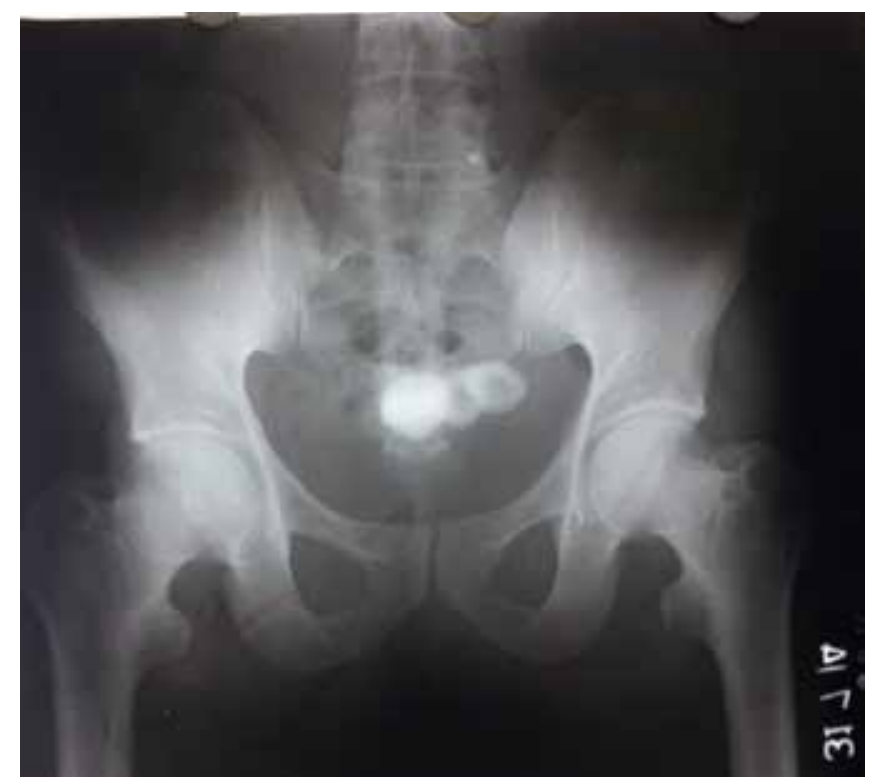

Fig.1: X-ray of KUB region showing multiple radio-opaque shadows in pelvic region.

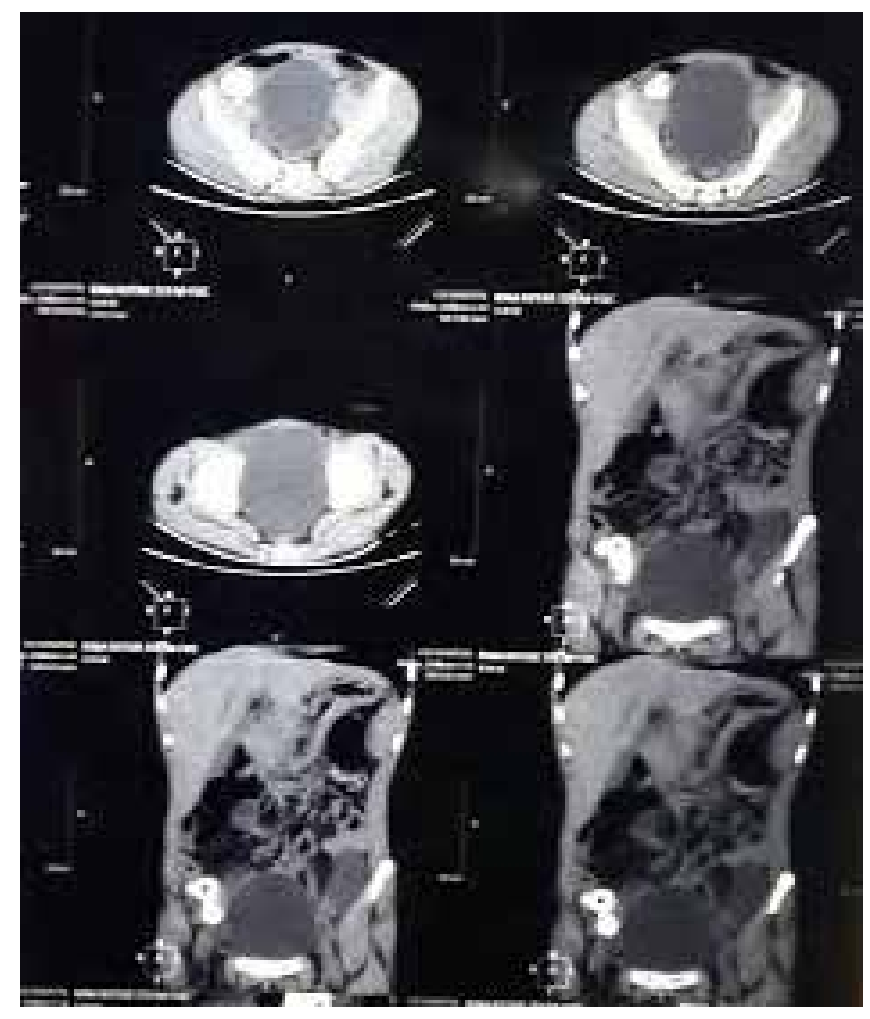

Fig.2: Non contrast screening CT shows opacity outside urinary bladder. 
[4]. However, calcification cannot occur without an alkaline $\mathrm{pH}$ shift, which normally occurs in the ileum [5], and hence, enteroliths in the distal small bowel are mainly composed of calcium salts which are less soluble in alkali and, therefore, tend to be precipitated in the distal small intestine [6] and are radio-opaque.

Stasis, as in diverticulae, or congenital/ acquired strictures, may also facilitate enzymatic hydrolysis of the biliary lecithin, favouring the appearance of free fatty acids that combine with calcium ions to form insoluble "calcium soaps" [7].

\section{Most enteroliths are asymptomatic,} complication if any, are likely to be severe such as obstruction, ileus, ulceration, melena, perforation $[3,7,8]$. However, our patient complained of intermittent spasmodic lower abdominal pain possibly due to intermittent obstructions due to the strictures of the ileum compounded by migrating enteroliths creating a "ball valve". Differential diagnoses of such calcified pelvic opacities include enteroliths, appendicolith, calculus, teratoma, calcified lymph nodes, multiple gallstones (gallstone ileus), foreign bodies, calcified fibroid, loose peritoneal bodies etc [3]. CT scan can give a provisional diagnosis.

Recommended treatment is the removal of the stone by enterotomy, excision of the diverticulum stricturoplasty and/or resection of the abnormal intestinal loop. Our patient underwent a resection and anastomosis because of multiple strictures in a short segment of the bowel.

\section{References}

1. Bery K, Chawla S, Gupta SK. Enteroliths and Intestinal Tuberculosis. Ind J Radiol Imag. 1985;39:225-230.

2. Van EHW, Sybrandy R. Enteroliths in a Meckel's

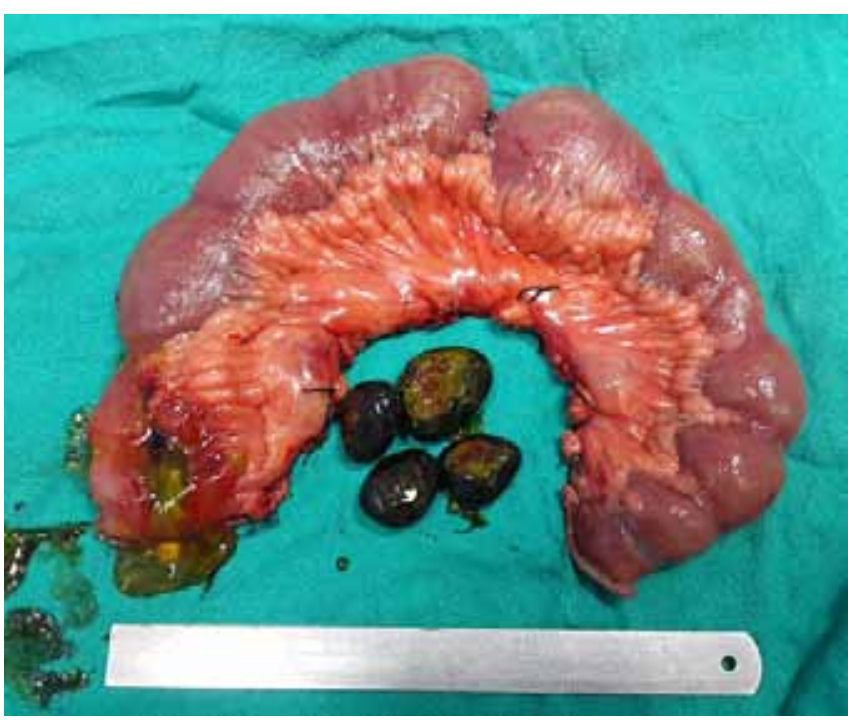

Fig.3: Enterolith with stricture of ileum.

diverculae. Radiology. 2000;2 14:524-526.

3. Sureka B, Singh V, Ranga A, Mittal MK. Giant migratory enterolith mimicking vesical calculus. Indian J Nephrol. 2014;24(6):402-403.

4. Lantsberg L, Eyal A, Khodadadi J, Hirsch M, Adear H. Enterolithiasis. J Clin Gastroenterol. 1988; 10:165-168.

5. Shocket E, Simon SA. Small bowel obstruction due to enterolith (bezoar) formed in a duodenal diverticulum: $A$ case report and review of the literature. Am J Gastroenterol. 1982;77:621 624.

6. Hayee B, Khan HN, Al-Mishlab T, McPartlin JF. A case of enterolith smallbowel obstruction and jejunal diveticulosis. World J Gastroenterol. 2003;9:883-884.

7. Crane PW, Crocker PR, Levison DA, Gilmore OJ. Enterolith ileus. J R Soc Med. 1988;81:2924.

8. Lantsberg L, Eyal A, Khodadadi J, Hirsch M, Adear H. Enterolithiasis. J Clin Gastroenterol. 1988; 10:165-168. 